\title{
Reconstruction of cascade-type neutrino events in KM3NeT/ARCA
}

\author{
D. Stransky ${ }^{* a, 1}$, T. Heid ${ }^{a, 2}$, C. James ${ }^{a, 3}$, J. Barrios-Martí ${ }^{b}$, L. A. Fusco ${ }^{c, d}$ and \\ A. Heijboer ${ }^{e}$ \\ for the KM3NeT Collaboration
}

${ }^{a}$ Friedrich-Alexander-Universität Erlangen-Nürnberg

Erlangen Centre for Astroparticle Physics, Erwin-Rommel-Str. 1, 91058 Erlangen, Germany.

E-mail:1 dominik. stranskyefau.de,

${ }^{2}$ thomas.heidefau. de,

${ }^{3} \mathrm{Clancy} \cdot$ james@physik.uni-erlangen. de

${ }^{b}$ Instituto de Física Corpuscular, IFIC (CSIC-UV)

Parque Científico, C/Catedrático José Beltrán, 2 E-46980 Paterna, Spain

E-mail: javier.barrios@ific.uv.es

${ }^{c}$ Dipartimento di Fisica e Astronomia dell'Università di Bologna, Viale Berti-Pichat 6/2, 40127, Bologna, Italy.

${ }^{d}$ INFN - Sezione di Bologna, Viale Berti-Pichat 6/2, 40127, Bologna, Italy.

E-mail: Ifuscoabo.infn.it

e Nikhef, Science Park, Amsterdam, The Netherlands

E-mail: aart.heijboer@nikhef.nl

KM3NeT is a future research infrastructure hosting the next-generation underwater neutrino observatory in the Mediterranean Sea. Within KM3NeT, the ARCA detector will be devoted to the observation of high-energy cosmic neutrinos both in diffuse and point source mode. The discovery of cosmic high energy neutrinos by the IceCube collaboration with a large fraction of shower-like events has led to the recognition of the particular importance of this channel at high(est) energies. While the neutrinos detected by IceCube are compatible with a diffuse distribution, good angular resolution is crucial for the identification of possible sources. Using Monte Carlo simulations, dedicated cascade reconstruction algorithms have been developed for KM3NeT/ARCA allowing for an efficient discrimination of showers from the main background of high energy atmospheric muon bundles and a high precision in the determination of the kinematic variables of the cascades. The optical properties of water and the new technology of KM3NeT with the multi-PMT digital optical module make it possible to reach an energy resolution of less than $10 \%$ and a median angular resolution of less than 2 degrees for high energy cascades taking place within the instrumented volume. The reconstruction methods and the results obtained are presented.

The 34th International Cosmic Ray Conference,

30 July- 6 August, 2015

The Hague, The Netherlands

* Speaker. 


\section{Introduction}

The search for high-energy cosmic neutrinos requires cubic-kilometre scale neutrino telescopes. With the discovery of cosmic high energy neutrinos by IceCube, it has been recognized that a large fraction of the neutrino events are of cascade type. This channel can thus also play an important role in finding high-energy neutrino sources. The main goal of KM3NeT [1] is to find these sources. Being in a first construction phase, the complete detector will in its final configuration consist of 6 detector blocks in the Mediterranean Sea with a total instrumented volume of about $3 \mathrm{~km}^{3}$.

As an intermediate step, the ARCA [2] detector will be built consisting of 2 blocks with a total instrumented volume of $1 \mathrm{~km}^{3}$. It has the dedicated goal to rediscover and investigate the diffuse cosmic neutrinos discovered by IceCube from the complementary field of view, and to search for possible sources. To find such sources, a high angular resolution in the reconstruction of such neutrino events is crucial, and it is possible thanks to the special design of the KM3NeT digital optical module (DOM) [3]. It consists of a pressure resistant glass sphere holding 31 3-inch PMTs mounted spherically. A standard ARCA block will comprise 115 vertical strings, each holding 18 of these DOMs. A detailed description of the KM3NeT detector layout can be found in these proceedings [4].

While a constant but small background noise is given by the decay of ${ }^{40} \mathrm{~K}$ in the sea, the main background for the detection of high energy cosmic neutrinos is given by the atmospheric muons and neutrinos. As opposed to cascades, muons produce a track signature in the detector. The atmospheric neutrino background also produces a high ratio of track-like to shower-like events as atmospheric muon neutrinos may interact via a charged current interaction in the vicinity of the detector, producing through-going muons. Therefore, to observe cosmic neutrinos in the cascade channel, a clear distinction between the manifestation of a track and a shower in the detector is necessary and achieved with the reconstruction algorithms presented here.

\section{Simulation chain}

The full simulation chain for KM3NeT/ARCA consists of four major stages: event generation, particle propagation, ${ }^{40} \mathrm{~K}$ noise simulation and PMT simulation including triggering.

For the event generation, a volume surrounding the instrumented volume, called can, is defined, such that events occurring outside the can produce insufficient light for significant detection. The can is chosen as a cylinder exceeding the instrumented volume by roughly $200 \mathrm{~m}$ in every direction.

Neutrino events of all flavours $\left(v_{e}, v_{\mu}, v_{\tau}\right.$, charged current - CC - and neutral current - NC) are generated with GENHEN [5]. This simulation package propagates neutrinos through the earth and forces them to interact in the vicinity of the detector. For neutrino interactions inducing showers only this corresponds to the can, whereas for interactions where a track is produced ( $v_{\mu} \mathrm{CC}$ and $v_{\tau}$ $\mathrm{CC}$ ) the lepton is propagated to the can in case the event occurred outside of it. The energy for the simulated neutrinos ranges from $10^{2}$ to $10^{8} \mathrm{GeV}$. As an example, Fig. 1 shows an event display for a simulated contained cascade induced by a $v_{e}$ of $1 \mathrm{PeV}$. 

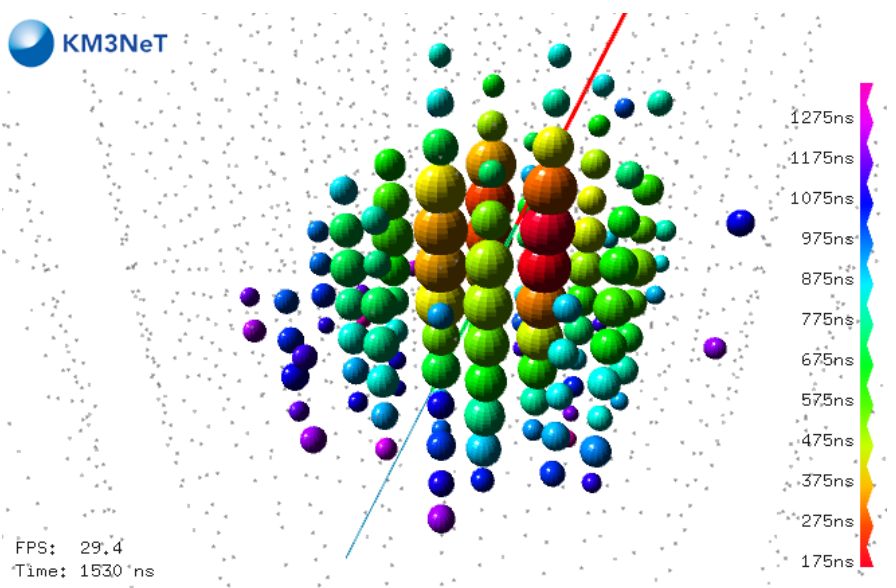

Figure 1: KM3NeT event display for a simulated contained $v_{e}$ $\mathrm{CC}$ event with a neutrino energy of $1 \mathrm{PeV} .{ }^{4} 0 \mathrm{~K}$ background hits are cut away by applying a cut on the signal strength in the DOMs. The scale on the right gives the hit times with respect to the time of the neutrino interaction.

\begin{tabular}{c|r|c} 
type & $N_{\text {sim }}$ & \multicolumn{1}{|c}{$T_{\text {live }}$} \\
\hline \hline atm. $\mu(E>10 \mathrm{TeV})$ & $18 \cdot 10^{5}$ & 3 months \\
atm. $\mu(E>50 \mathrm{TeV})$ & $80 \cdot 10^{5}$ & 3 years \\
\hline$\left(v_{e}+\bar{v}_{e}\right) \mathrm{CC}$ & $14 \cdot 10^{5}$ & \\
$\left(v_{\mu}+\bar{v}_{\mu}\right) \mathrm{CC}$ & $8 \cdot 10^{5}$ & \\
$\left(v_{e, \mu}+\bar{v}_{e, \mu}\right) \mathrm{NC}$ & $24 \cdot 10^{5}$ & $>10^{4}$ years \\
$\left(v_{\tau}+\bar{v}_{\tau}\right) \mathrm{CC}$ & $120 \cdot 10^{5}$ & \\
$\left(v_{\tau}+\bar{v}_{\tau}\right) \mathrm{NC}$ & $100 \cdot 10^{5}$ &
\end{tabular}

Table 1: Rounded number of simulated events and corresponding live time. The production for atmospheric muons is separated in two parts with different low energy thresholds. For each group of neutrino types the number of simulated events is equality distributed among them. For the neutrinos the given live time holds for energies above $10^{4} \mathrm{GeV}$.

Atmospheric background is simulated with MUPAGE [6, 7] and a CORSIKA production to estimate the 'self-veto' effect [8,9]. In addition, the optical properties of seawater [5, 10], background hits from K40 [11], the response of the PMTs [3], and data acquisition and event identification [12] is fully accounted for. Triggered PMT hits are saved as hit time with the corresponding measured charge translated into a Time over Threshold (ToT).

The number of events generated for each neutrino flavour and interaction type and the atmospheric background are summarized in table 1 together with the corresponding livetime.

\section{Reconstruction algorithms}

Three dedicated shower reconstruction algorithms exist for KM3NeT/ARCA, which are in the following called reconstruction method 1-3, respectively. Reconstruction method 1 is a reconstruction algorithm specifically developed for KM3NeT taking full advantage of the features of the multi-PMT DOM. It shows the best performance among the three algorithms regarding energy and direction resolution for cascade events taking place in the detector. The other two were originally developed for the ANTARES experiment in the SeaTray [13] framework and adopted to the new detector layout of ARCA. These algorithms include the calculation of parameters that allow for the determination of the event topology. As most of the background events for an experiment like 

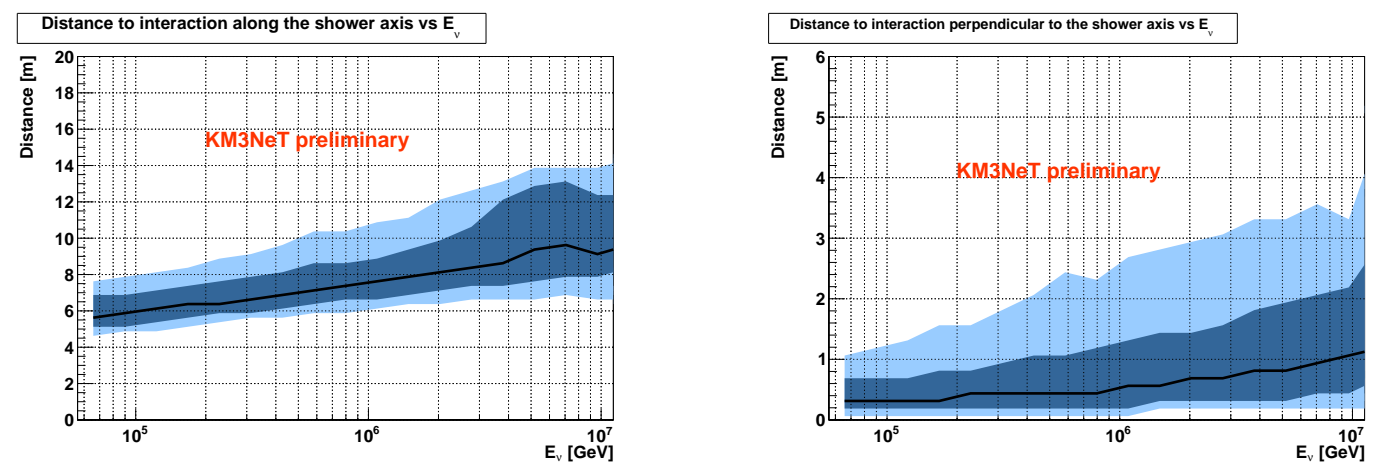

Figure 2: Vertex resolution along the shower direction (left) and perpendicular to the shower direction (right) after an event selection cut according to an ARCA sensitivity analysis [7], leaving dominantly contained showers. In dark and light blue the $1 \sigma$ and $90 \%$ bands are shown, respectively.

KM3NeT are of track-type, it is possible to reduce this background by using these parameters [7]. In the following, resolution plots will only be shown for reconstruction method 1, while the description of results of the other two also deals with their performance discriminating shower and track.

\subsection{Reconstruction method 1}

Reconstruction method 1 has been specifically developed for the precise reconstruction of the energy and direction of cascades taking place within the instrumented detector volume. While taking advantage of the features of the KM3NeT multi-PMT DOM, the hit selection is designed to be simple and to account for a fast reconstruction. Therefore, hits occurring in a time window of $350 \mathrm{~ns}$ on the same PMT are merged into a single hit having the time of the first hit. After merging, coincidences are selected as two merged hits occurring on a single DOM within $20 \mathrm{~ns}$ on different PMTs. These coincidences are used for the vertex fit, which is an M-estimate minimization of the residuals. The residuals are computed assuming a spherically expanding light shell from a single point, the assumed shower position. As a result, the position and time fitted are shifted from the neutrino interaction vertex in the direction of the shower to the shower maximum. This can be seen on the left panel of Fig. 2 which shows the vertex resolution as a function of the neutrino energy along the shower direction. In the right panel the distance of the reconstructed position perpendicular to the interaction vertex is shown. The vertex resolution on the shower maximum thus reaches a precision well below $0.5 \mathrm{~m}$ in the important energy regime.

For the direction and energy reconstruction a likelihood-fit method is used, also based on the above mentioned hit merging. The concept of the method is based on counting the number of hit PMTs in a DOM. In a DOM near the position of a high energy shower maximum, all PMTs will be saturated, but going further away from the shower, some PMTs facing away are not hit. At larger distances, only a few PMTs facing the shower are hit. Additionally as most light from the cascade is emitted under the Cerenkov angle, for DOMs at a given distance there is an angular dependence upon the number of PMTs hit. Based on this information, a three dimensional probability density function (PDF) is derived from MC simulations. The PDF gives the number of hits as a function of three parameters: the distance of the shower position to the centre of the hit DOM; the angle between the shower direction and a vector pointing from the shower to the DOM; and the direction 

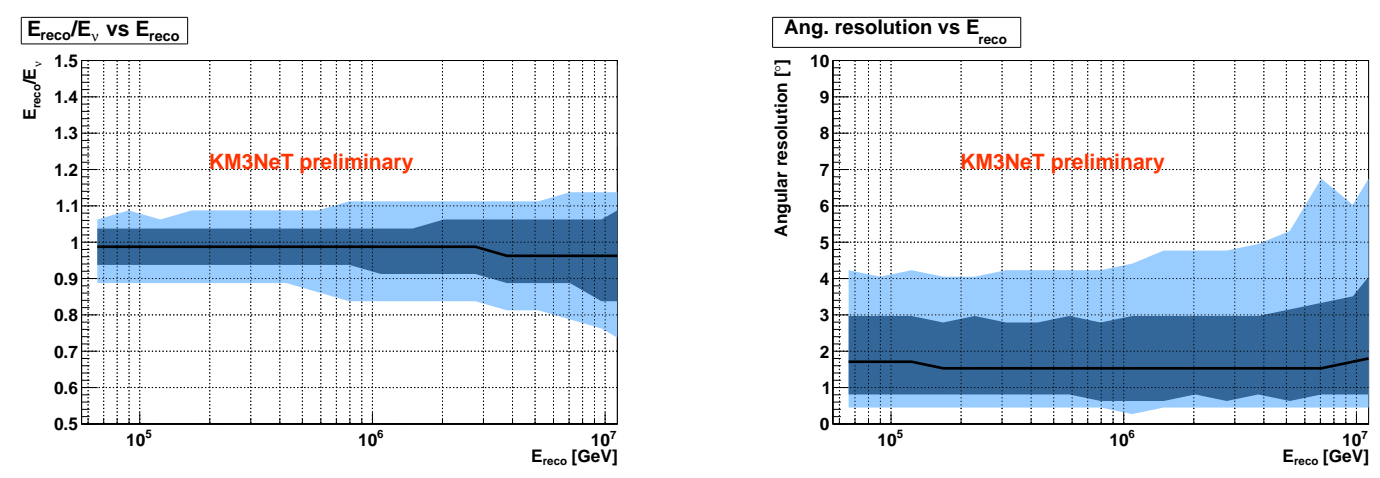

Figure 3: Energy resolution (left) and angular resolution (right) after an event selection cut according to an ARCA sensitivity analysis [7]. In light and dark blue the $1 \sigma$ and $90 \%$ quantile bands are shown, respectively.

of the hit PMT in the DOM. This PDF has a linear dependency on the shower energy. In the end, the mean expected number of background hits in a time window covering the whole event is then added to get the expected total number of hits. Using the Poisson probability to observe zero hits $P_{0, i}=\exp \left(-\mu_{i}\right)$ with the mean expected total number of hits $\mu_{i}$ in PMT $i$, the likelihood is then defined as

$$
\log L=\sum_{i}^{\text {empty PMTs }} \log \left(P_{0, i}\right)+\sum_{i}^{\text {hit PMTs }} \log \left(1-P_{0, i}\right)
$$

where the first sum is over all non hit PMTs and the second sum over all PMTs that have at least one hit. The likelihood is fitted via the shower direction and energy.

The energy resolution achieved with this method is roughly $10 \%$ and the mean angular error is roughly $2^{\circ}$ for contained cascade events. After an appropriate event selection the resolution can even be enhanced. Figs. 3 show the achieved energy and direction resolution in the energy range most important for ARCA ( $E \gtrsim 50 \mathrm{TeV})$ assuming the event selection shown in Ref. [7]. The energy resolution is thus even in the range of $5 \%$ and the mean angular error is roughly $1.5^{\circ}$.

\subsection{Reconstruction method 2}

Reconstruction method 2 is based on four consecutive steps of shower hit selection and fit, under the assumption that Cherenkov light is emitted spherically from a point source located close to the shower maximum. Before starting this procedure, a preselection of hits is made on the basis of the applied trigger algorithms. The first step finds a first guess of the vertex position by taking the mean space-time position of hits. Using this first guess, the second step consists of a hit selection by searching for causal correlations of the hits with respect to the first guess. In the third step, the final vertex fit is performed based on time residual minimization. During that step, important parameters quantifying the quality of the fit are computed.

The energy and direction are estimated in the last step, taking into account the final vertex fit. The energy is computed from the ToT distribution of the selected hits in the detector and the direction is estimated from the mean light direction of all selected hits. With this method a mean vertex resolution of roughly 6 metres and an energy resolution of less than 0.4 in the logarithm of the energy is achieved. 


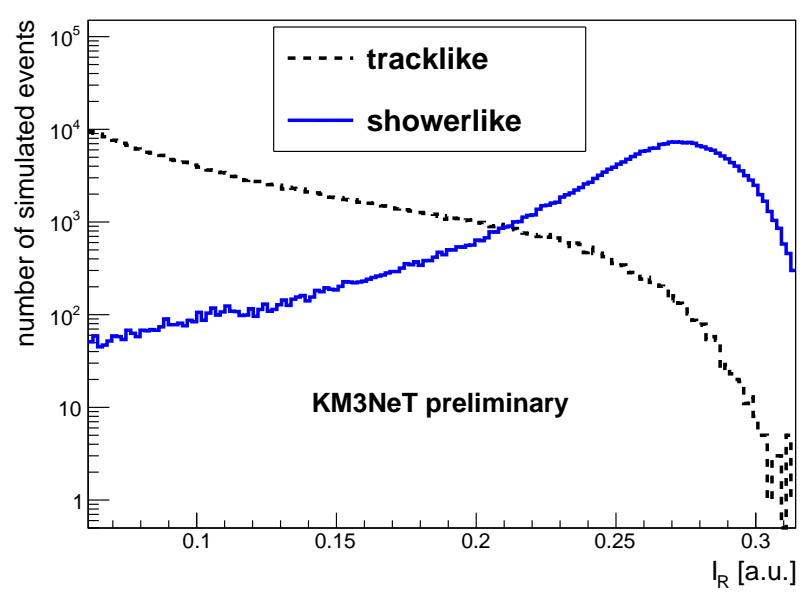

Figure 4: The distribution of simulated track (black, dashed) and shower events (blue, solid) versus the Inertia Ratio $I_{\mathrm{R}}$.

The hits from the final selection are also used to produce a quantity named Inertia Ratio $I_{R}$, described here as an example of parameters for track versus shower discrimination. $I_{\mathrm{R}}$ is a measure of the three dimensional hit distribution in the detector analogous to the tensor of inertia of an extended system of mass points: for a shower taking place in the detector, the hit distribution is approximately spherical as opposed to the hit distribution produced by e.g. an atmospheric muon track. Fig. 4 shows the discrimination power of $I_{\mathrm{R}}$. Together with other reconstructed parameters, it is used in ARCA sensitivity analysis [7] and is involved in the event selection used for Figs. 2 and 3 .

\subsection{Reconstruction method 3}

Reconstruction method 3 is based on maximum likelihood approaches for the vertex, energy and direction reconstruction. The algorithm begins with a cleaning of the hit sample by choosing only hits with large amplitude to suppress background from ${ }^{40} \mathrm{~K}$. Thereafter, hits coincident with those selected in the first step are added to the hit sample. The earliest hit of this selection is taken as a first guess of the vertex position and time. A simple scan fit is then performed to improve this guess. With the help of the fitted vertex a further cleaning of the hit sample is performed by applying a time residual cut. To reduce ambiguities in the reconstruction, further minimum constraints on the hit sample are applied.

The final sample of selected hits is used to perform a final reconstruction of the vertex in one step and the energy and direction reconstruction in another step. For both steps a maximum likelihood method is performed based on PDFs generated from Monte Carlo simulations. The PDF for the vertex fit has two parameters: the distance between the interaction vertex and the DOM, and the time for a photon to propagate to the DOM. The PDF for the energy and direction reconstruction is a function of three parameters: the light emission direction with respect to the initial neutrino direction, the amount of light received by the PMT under the assumption of isotropic light emission and the shower energy. For the maximization of the likelihood the Gulliver [13] package in SeaTray is used. With this method a mean vertex resolution of 5 metres, an energy resolution of roughly $20 \%$ and a direction resolution of 5 degrees can be achieved. 


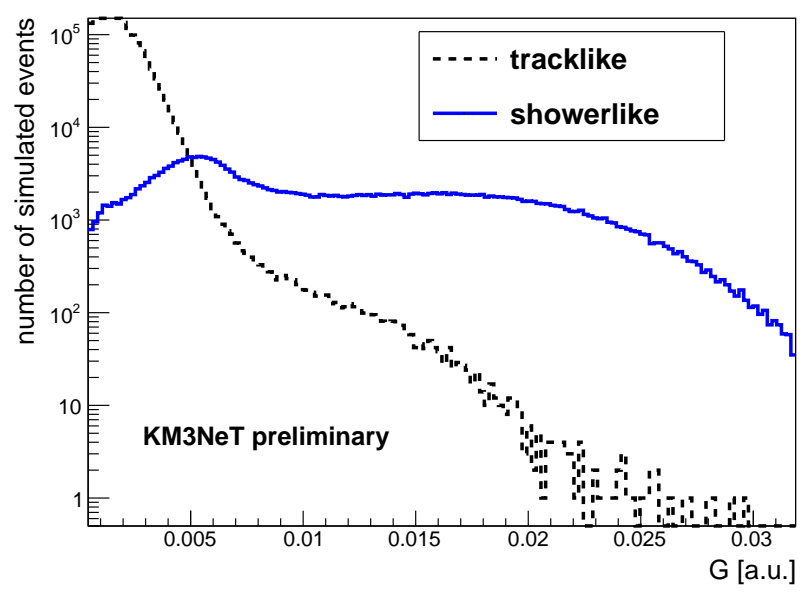

Figure 5: The distribution of simulated track (black, dashed) and shower events (blue, solid) versus the $G$ parameter.

Quality parameters that are useful in distinguishing shower-like from track-like events are computed by this algorithm and are used in ARCA sensitivity analyses [7]. An example of these parameters is the $G$ parameter. It is calculated from the time residuals as an exponential weighted sum:

$$
G=\frac{1}{N} \sum_{i}^{N} \mathrm{e}^{-\frac{\left(t_{\mathrm{r}, i} / \mathrm{ns}\right)^{2}}{4.5}},
$$

where $N$ is the number of selected hits and $t_{\mathrm{r}, i}$ is the time residual of the $i$-th hit. The factor of 4.5 in the denominator of the exponent accounts for the time resolution of the DOM. This parameter thus measures the agreement of the time residual distribution to the shower isotropic emission assumption, where the exponential weighting suppresses the impact of outliers. Fig. 5 shows the discrimination power for the $G$ parameter.

\section{Conclusion}

With the cascade reconstruction algorithms developed for KM3NeT/ARCA exploiting the features of the new KM3NeT technology, precise determination of the kinematic variables of a cascade occurring inside the instrumented volume is possible. For the best performing reconstruction method the angular resolution is better than $1.5^{\circ}$ while an energy resolution of roughly $5 \%$ can be achieved.

In addition, reconstruction parameters from the presented algorithms presented allow for a precise discrimination between tracks and showers and the identification of signal events over the background. During one of the ARCA sensitivity analysis [7], an event selection is made based on the reconstruction parameters leaving dominantly signal events. Also events where the Monte Carlo vertex lies outside the instrumented volume survive this selection. In Fig. 6 the energy and angular resolution as a function of energy for especially these events is shown. It thus can be seen that even for such events outside the instrumented volume a very well precision on the determination of the kinematic variables is possible. 

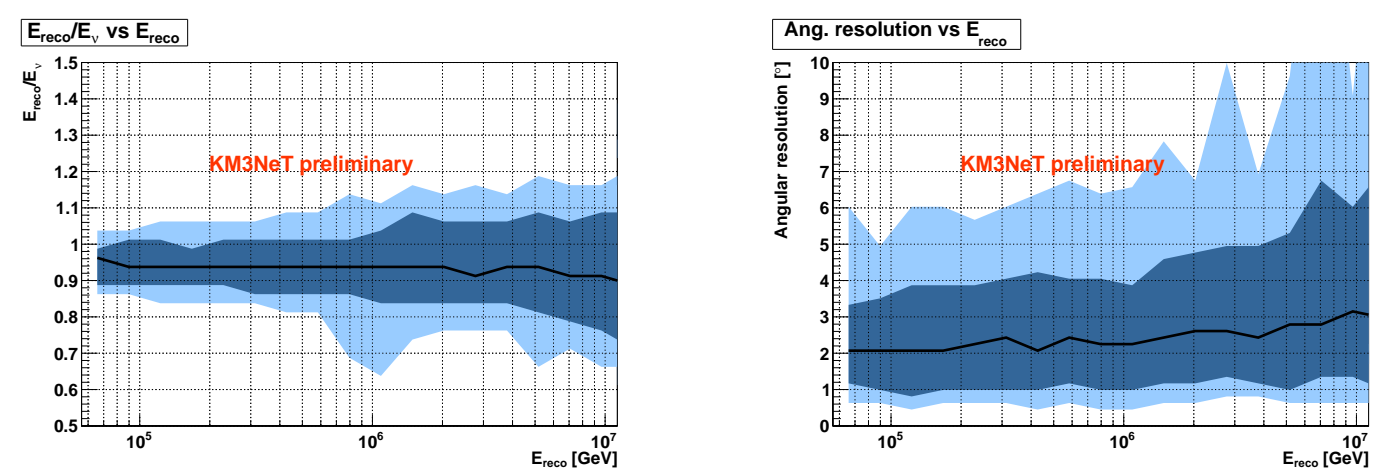

Figure 6: Energy (left) and angular resolution (right) for events outside the instrumented volume after an appropriate selection cut procedure (see [7]). In dark and light blue the $1 \sigma$ and $90 \%$ quantile bands are shown, respectively.

\section{References}

[1] www.km3net.org.

[2] P. Piatelli et al., for the KM3NeT Collaboration, proceedings of this conference ID 1158.

[3] R. Bruijn et al., for the KM3NeT Collaboration, proceedings of this conference ID 1157.

[4] C. James et al., for the ANTARES/KM3NeT Collaborations, proceedings of this conference ID 24.

[5] A. Margiotta et al., for the ANTARES Collaboration, Nuclear Inst. and Methods in Physics Research, A, Volume 725, p. 98-101.

[6] Y. Becherini, et al., Astroparticle Physics 25 (2006) 1; G. Carminati, et al., Computer Physics Communications 179 (2008) 915.

[7] D. Stransky et al., for the KM3NeT Collaboration, proceedings of this conference ID 1107.

[8] D. Heck et al., (https://www.ikp.kit.edu/corsika/), Wissenschaftliche Berichte, Forschungszentrum Karlsruhe FZKA 6019 (1998).

[9] T. Heid et al., for the KM3NeT Collaboration, proceedings of this conference ID 1067.

[10] G. Riccobene et al., Astropart. Phys. 27 (2006) 1.

[11] C. Hugon et al., for the KM3NeT Collaboration, proceedings of this conference ID 1106.

[12] S. Biagi et al., for the KM3NeT Collaboration, proceedings of this conference ID 1172.

[13] C. Kopper, A software framework for km3net, Nucl. Instrum. Meth. A 602 (2009) 107-110. 